\title{
NIKLAS LUHMANN NO BRASIL: RESISTÊNCIAS ACADÊMICAS AO ESTUDO DA TEORIA DOS SISTEMAS
}

David Barbosa de Oliveira ${ }^{1}$ Vinicius Madureira Maia ${ }^{2}$

\section{NIKLAS LUHMANN AND SOCIOLOGY IN BRAZIL: RESISTANCE TO THE STUDY OF SYSTEMS THEORY}

RESUMO: O presente trabalho tem por intento uma breve discorrência acerca de Niklas Luhmann, sua obra e também da crítica a esta, ao mesmo passo em que se conjectura três motivos para a sua aventada irreceptibilidade no cenário acadêmico brasileiro - e não apenas brasileiro - , mesmo vinte anos após sua morte em 1998. A pesquisa é de fundo eminentemente bibliográfico com vistas a uma reflexão acerca da importância e, sobretudo, da rejeição do sociólogo em questão, a uma análise menos sujeita à acrisia que acompanha, o mais das vezes sem uma percepção de limites, a importação de modelos teóricos construídos em outros ambientes, especialmente na Alemanha.

Palavras-chave: Niklas Luhmann. Teoria dos Sistemas. Sociologia. Filosofia. Crítica.
ABSTRACT: One has the purpose to discuss in this work about Niklas Luhmann, his work and his criticism as well, focusing on three hypotheses for his non reception in the Brazilian (and not just the Brazilian) academic scene, in spite of the two decades, this year, since his death. An eminently bibliographical research is developed in order to achieve a reflection regarding the German sociologist in question, an analysis that intends to be less subject to the acrisia, which accompanies the importation of theoretical models developed in other environments, particularly in Germany.

Keywords: Niklas Luhmann. Systems Theory. Sociology. Philosophy. Criticism.

\footnotetext{
${ }^{1}$ Professor da Universidade Federal do Ceará (UFC).

${ }^{2}$ Mestrando em sociologia na Universidade Estadual do Ceará.
} 


\section{INTRODUÇÃO}

Este trabalho, subsidiado por extensa revisão de literatura, tem por intento discorrer brevemente acerca de Niklas Luhmann, sua obra e também sobre a crítica com relação a ela, ao passo em que se conjectura três motivos para a sua aventada não receptibilidade no cenário acadêmico. Para tanto, por meio de uma pesquisa eminentemente bibliográfica, busca uma reflexão acerca da importância e, sobretudo, da rejeição do sociólogo em questão, uma análise menos sujeita à acrisia que acompanha - o mais das vezes sem uma percepção de limites - a importação de modelos teóricos construídos em outros ambientes, especialmente na Alemanha.

Desse modo, o artigo para além de busca entender a importância de Luhmann no campo da sociologia, busca apontar as oposições a teoria dos sistemas. As principais resistências trazidas no texto são: primeiro, a de que essa teoria seria uma espécie de herdeira radical do positivismo, reduzida a uma sociologia conservadora de tendência tecnocrata; segundo, a crítica de que houvera colocado o homem fora da sociedade, posto que o homem seria ambiente da sociedade; e, por fim, o grau abstrato de seu artefato teórico, haja vista que, segundo ele, a realidade não se capta e nem se entrevê senão por meio de conceitos laboriosos.

A hipótese deste trabalho é de que algumas críticas de acadêmicos e interlocutores fomentaram resistências para uma assimilação mais profícua no Brasil. Assim, buscamos compreender, neste artigo, os pressupostos teóricos que impelem Luhmann a criar a teoria dos sistemas, bem como, a partir de algumas críticas, buscamos compreender como esse autor permanece carente de pesquisadores que dialoguem com sua teoria.

\section{2 À ESPERA DE NIKLAS LUHMANN}

A despeito de sua fértil produção acadêmica, que se traduz em um número de publicações ainda à espera de serem listadas por completo, e que entremostram a sua orientação, ainda que por recorte, à confecção de uma pesquisa abrangente do social, o sociólogo alemão Luhmann - um dos autores tido como dos mais interessantes no âmbito 
da sociologia do direito contemporânea ${ }^{3}$ - e a sua Teoria dos Sistemas parecem penetrar ainda infimamente nos estudos dos programas de pós-graduação em sociologia brasileiros atuais: "não adquiriu toda a notoriedade que sua envergadura propõe, e seus comentadores e intérpretes ainda são escassos no Brasil. É, no entanto, uma teoria instigante e que pode ser incluída nos trabalhos mais importantes da área da sociologia, no século passado" (NEVES, 2004, p. 122).

O empreendimento de uma pesquisa abrangente do social consubstancia o tour de force de Luhmann, o qual tomava por baldada a perspectiva que, a partir de certo sistema ou subsistema, tenha por pretensão (a)firmar-se como ponto arquimédico de uma observação estrita da sociedade, sobretudo a assim chamada pós-moderna, ou "hipercomplexa", "policontextural", no jargão corrente de dois de seus interlocutores, Marcelo Neves e Gunther Teubner (ambos juristas, o que é significativo), respectivamente. Sua relevância residiria precisamente em reconhecer que a complexidade desta mesma sociedade torna infrutífero qualquer modelo simplista (socialmente inadequado, porquanto não submisso ao crivo da multicentridade atual) que intencione descrevê-la, esclarecê-la, quiçá justificá-la "a partir de um único mecanismo ou aspecto: o poder, no caso de Michel Foucault, a luta de classes, de Karl Marx, o capital simbólico, de Pierre Bourdieu, ou o agir comunicativo, de Jürgen Habermas" (NEVES, 2013, p. 342).

Isso significa, em primeiro lugar, que a diferença entre sistema e ambiente desenvolve-se em diversos âmbitos de comunicação, de tal maneira que se afirmam distintas pretensões contrapostas de autonomia sistêmica. E, em segundo lugar, na medida em que toda diferença se torna "centro do mundo", a policontexturalidade implica uma pluralidade de autodescrições da sociedade, levando à formação de diversas racionalidades parciais conflitantes. Falta, então, uma diferença última, suprema, que possa impor-se contra todas as outras diferenças. Ou seja, não há um centro da sociedade que possa ter uma posição privilegiada para a sua observação e descrição; não há um sistema ou mecanismo social a partir do qual todos os outros possam ser compreendidos ${ }^{4}$ (NEVES, 2009, p. 23-24).

\footnotetext{
${ }^{3}$ Tércio Sampaio Ferraz Jr. (1980, p. 01) comenta que o enfoque empírico desse importante autor se poderia distinguir dos demais pelo menos por três razões básicas: "Em primeiro lugar, porque afronta de modo explícito a questão teórica da definição do direito [...]. Em segundo lugar, porque propõe uma análise dos problemas precípuos da sociologia do direito a partir de uma perspectiva unitária e bastante original. Em terceiro lugar, porque amplia esta perspectiva não só dentro de uma concepção global da teoria sociológica, mas também de ângulo epistemológico e até de uma filosofia geral [...]".

${ }^{4}$ Ver ainda: "Para Luhmann, não só o mundo não é um sistema, mas, além disso, não há nenhum sistema que possa abarcar todos os aspectos da complexidade do mundo. Por seu turno, na sociedade moderna, nenhum sistema, e
} 
Em não havendo, pois, um topos que se sustente como um tipo de panóptico da sociedade hipercomplexa - dado que toda observação seria parcial, sob o ponto de vista sistêmico -, "nem mesmo a política é um centro ou um lugar privilegiado da sociedade, mas um sistema em concorrência com os outros" (NEVES, 2009, p. 25). Assim, com a exclusão, pela autonomia dos sistemas funcionais, de uma metarregulação por códigos e programas de um sistema prevalente, a asserção clássica contraposta, "ao por a política no centro como supersistema da sociedade moderna, só tem levado a desilusões" (NEVES, 2009, p. 25; NEVES, 2012, p. 67) ${ }^{5}$.

A partir daí, poder-se-ia arriscar, de pronto e à moda de suspeita, três grandes razões para o desinteresse geral em relação a Luhmann, sem nenhuma razão para supor que se trataria de uma lista arbitrariamente completa ou exaustiva, nem tampouco que esta será doravante abordada de forma definitiva.

A primeira: talvez ainda pese sobre a Teoria dos Sistemas a generalização de um juízo prematuro de que ela seria, em virtude de sua desconcentração do sistema político, uma espécie de herdeira radical do positivismo, reduzida a uma sociologia conservadora de tendência tecnocrata ${ }^{6}$. Esse pré-conceito se deparou mais fervescente a princípio, quando da publicação, em 1971, do debate "Theorie der Gesellschaft oder

nenhum saber, poderá ocupar o lugar de um arqui-sistema, como acontece com a política, no caso paradigmático da koinonia politikê aristotélica" (SANTOS, 2005, p. 10, itálico no original).

${ }^{5}$ Contrariamente a Luhmann, Friedrich Müller sustenta: “A política realmente não é, como nas teorias de Luhmman ou Habermas, simplesmente um subsistema da sociedade. Luhmann, mais do que todos, quer limitar a política ao mais estreito sistema político. Com isso, ele a exclui dos outros "subsistemas" da sociedade e, desse modo, os despolitiza de forma marcante. A sociedade não é uma totalidade prévia e objetiva. Ela aparece, muito mais, como resultado complexo e instável de contínuos esforços e ações. A política alcança um significado fundamental que ultrapassa o aparato e órgãos estatais. Ela representa a própria base de toda sociabilidade. Não está vinculada a um determinado sistema funcional, mas eclode quanto (nos conceitos da teoria dos sistemas) o "fechamento" sistêmico colapsa. Sempre, então, que uma decisão "absoluta", "definitiva", "clara" (que deveria surgir de um centro indiscutível, que, no entanto, não existe) não seja possível. Momentos de poder e decisão existem em todas as regiões sociais. Daí que a política não se deixe limitar a uma "política da sociedade" no sentido luhmanniano e, por isso, um primado da política para a sociedade moderna não é destrutivo, como teme esse teórico" (MÜLLER, 2010, p. 282).

6 "It [theory of social systems] has been regularly misread as conservative, overly structural, positivist and disconnected from other contemporary theories; especially the ones influenced by post-structuralism, gender theory, postcolonialism, and spatial and embodied understandings of society. Such misunderstandings help to explain why, particularly in the English-speaking world, reception of Luhmann's work has been reluctant and uneven. While there is no paucity of introductions, general discussions and handbooks on Luhmann's work, some of the misattributions and ossified readings persist. Part of the problem is the lack of fruitful critical dialogues between Luhmann's theory and other theoretical perspectives that would manage to set Luhmann in a new light, away from received readings and originary orthodoxies, and in line with contemporary theoretical developments" (PHILIPPOPOULOS-MIHALOPOULOS; COUR, 2013, p. 01). 
Sozialtechnologie - was leistet die Systemforschung?", 7 travado entre Habermas e

Luhmann, com réplicas e tréplicas, seguindo-se doravante um acalorado debate frutífero

quase trintenial (NEVES, 2013, p. 342). Posta a interrogação assim à guisa de dicotomia e

polaridade em termos políticos, Teoria da Sociedade versus Tecnologia Social, insinua-se

que Habermas seria o pretenso defensor da primeira, e Luhmman, da segunda.

Na minha aceitação, em 1969, na então criada Faculdade de Sociologia da Universidade de Bielefeld, em 1969, vi-me confrontado com a solicitação de nomear o projeto de pesquisa no qual eu trabalho. Meu projeto se designava à época e desde então: Teoria da Sociedade; Duração: 30 anos; Custos: nenhum. As dificuldades do projeto, no que tange à duração, foram avaliadas de modo realístico. A literatura sociológica disponível naquele tempo oferecia poucas pistas para assegurar que um tal projeto fosse sequer possível. Não era esse o único óbice, pois a ambição de uma Teoria da Sociedade foi também bloqueada por obstáculos neomarxistas. Pouco depois, um volume de uma discussão travada com Jürgen Habermas foi lançado sob o título: "Teoria da Sociedade ou Tecnologia Social. O que realiza a pesquisa sistêmica?". A ironia deste título consiste em que nenhum dos autores tinha por desígnio a defesa de uma Tecnologia Social. Havia divergências, todavia, sobre como uma Teoria da Sociedade devia parecer; e é ainda sintomaticamente significativo que o lugar de uma Teoria da Sociedade não tenha sido tomado, aos olhos do público, inicialmente por meio de uma teoria, mas de uma polêmica (LUHMANN, 1997, p. $11)^{8}$.

Vê-se que, na cena acadêmica alemã, a teoria sistêmica se assimilava como apenas mais um item do ideário burguês e outro lado de sua moeda ideológica. Não significa,

\footnotetext{
7 "[...] in order to affirm the concept of reduction, Luhmann provoked all intellectuals. This was probably the secret reason why the book from Suhrkamp publishing house, published jointly by Luhmann and Habermas (1971), got the title Theorie der Gesellschaft oder Sozialtechnologie [Theory of society or social technology]. In an interview, Luhmann stated that he had never wanted to be a social technologist. This is why everyone was provoked because until then, the concept of reduction represented to all intellectuals the embodiment of absolute evil. Nothing could be worse than reducing or being reductive. When the concept was suddenly affirmed - although it had not been affirmed as selfdifference, but rather as an external reference - all alarms went off" (GUMBRECHT, 2012, p. 07). Detalhe para "all alarms went off" ["todos os alarmes dispararam"] que, em inglês, é uma expressão de multifário uso: soam ante situação de perigo ou de risco (alarmar, por em alarme, alarmante, etc.], um apelo às armas; para avisar trivialmente quanto a um compromisso ou dever; ou ainda para despertar quem porventura dorme - ou sonha, "acordar para a vida".

${ }^{8}$ No original: “Bei meiner Aufnahme in die 1969 gegründete Fakultät für Soziologie der Universität Bielefeld fand ich mich konfrontiert mit der Aufforderung, Forschungsprojekte zu benennen, an denen ich arbeite. Mein Projekt lautete damals und seitdem: Theorie der Gesellschaft; Laufzeit: 30 Jahre; Kosten: keine. Die Schwierigkeiten des Projekts waren, was die Laufzeit angeht, realistisch eingeschätzt worden. Die Literaturlage in der Soziologie bot damals wenig Anhaltspunkte dafür, ein solches Projekt überhaupt für möglich zu halten. Dies nicht zuletzt deshalb, weil die Ambition einer Theorie der Gesellschaft durch neomarxistische Vorgaben blockiert war. Der kurz darauf veröffentlichte Band einer Diskussion mit Jürgen Habermas trug den Titel: Theorie der Gesellschaft oder Sozialtechnologie. Was leistet die Systemforschung? Die Ironie dieses Titels lag darin, daß keiner der Autoren sich für Sozialtechnologie stark machen wollte, aber Meinungsverschiedenheiten darüber bestanden, wie einer Theorie der Gesellschaft auszusehen habe; und es hat symptomatische Bedeutung, daß der Platz einer Theorie der Gesellschaft in der öffentlichen Wahrnehmung zunächst nicht durch eine Theorie, sondern durch eine Kontroverser eingenommen wurde."
} 
todavia, que o solapamento do primado atribuído amiúde à política tome, na teoria Iuhmanniana, o sistema político por irrelevante. Pelo contrário (BORSCH, 2011, p. 115). Em uma entrevista voltada à mitigação desse receio expresso à época (considerando o contexto europeu da Guerra Fria, sobretudo o ambiente intelectual de uma Alemanha ainda em "acerto de contas com o passado" [Vergangenheitsbewältigung] mas, em parte, sob domínio soviético) de que a teoria dos sistemas, tal como formulada, enquanto nova ideologia de uma sociedade tecnocrática, poderia justificar e estabilizar toda forma de relações de poder existentes, nomeada e sub-repticiamente os regimes totalitários porque tida por abstêmia de padrões específicos para a racionalidade e a irracionalidade de certa ordem social $-{ }^{9}$, Luhmman contemporiza que sua pesquisa seria, sim, desprovida de barreiras imanentes contra o uso ou abuso políticos ("Wie sollten solche Sperre auch aussehen?!", ele indaga, curioso), tergiversando, contudo, que o controle político e o problema da estabilização ou não da autoridade devem, antes, vislumbrar-se concreta e detalhadamente no âmbito do próprio sistema político, por seus respectivos atores, uma vez que "os cientistas", conclui ele de modo irônico, "não podem estar em Bonn todo santo dia"10.

Um tal arremate, assim espirituoso, bem se entende com a própria concepção do fazer sociológico luhmanniano e é a clave mesma para a sua compreensão e divisa, cuja posição científica evoca o postulado weberiano da liberdade em relação a juízos de valor (Werturteilfreiheit, a "neutralidade axiológica" do conhecimento histórico-social).

Seguramente, é uma busca incessante a minha tentativa de manter distância em relação a tais fenômenos, com os quais uns se exaltam ou para os quais normalmente se requer engajamento emocional ou normativo. Meu objetivo-mor

\footnotetext{
9 "Es gehört zu den gängigen Annahmen, dass es so etwas wie eine ,Kritische Systemtheorie' nicht gibt. Systemtheorie sei nicht kritisch-emanzipativ, sondern als rein deskriptive Beobachtungsform die "Hochform eines technokratischen Bewusstseins", eine "Apologie des Bestehenden um seiner Bestanderhaltung willen". So hat Jürgen Habermas in der Debatte mit Niklas Luhmann formuliert und diese Charakterisierung hat die kritische Theorie in normativer Hinsicht Lange Zeit gegenüber der Systemtheorie hermetisch-autopoietisch verschlossen" (FISCHER-LESCANO, 2013, p. 13-14). Tradução livre: "É próprio às suposições triviais que algo como uma "teoria dos sistemas crítica" não existe. A teoria dos sistemas, diz-se, não é crítico-emancipatória; trata-se antes de um modo de observação puramente descritivo da "forma suprema de uma consciência tecnocrática", uma "apologia do status quo em prol de sua conservação". Assim formulou Habermas em seu debate com Niklas Luhmann, e, por um longo tempo, essa caracterização tem fechado, em termos normativos, hermética e autopoieticamente a teoria crítica para a influência da teoria dos sistemas."

${ }^{10}$ LUHMANN, 1973. A entrevista "Gespräch mit Ulrich Boehm" se encontra disponível integralmente no sítio: http://www.youtube.com/watch?v=qRSCKSPMuDc. Acesso em: 01 fev. 2018. *Bonn: antiga capital administrativa da Alemanha, de 1949 até a reunificação em 1990.
} 
como cientista é o melhoramento da descrição sociológica da sociedade e não o melhoramento da sociedade. $O$ que naturalmente não exclui o atuar como um outro, ao se tomar parte na vida política ou eclesiástica ou ainda em ocasiões de cunho artístico (LUHMANN, 1992b, p. 02) ${ }^{11}$.

O melhoramento da descrição sociológica da sociedade enquanto objetivo principal de Luhmann (e não o melhoramento da sociedade em si) é oriundo de sua concepção da incapacidade da sociologia, ${ }^{12}$ na condição de ciência empírica, de explicar adequadamente a realidade social: "ela não possui - pode-se dizer de maneira bastante rigorosa nenhum verdadeiro conhecimento garantido acerca da nossa sociedade" (LUHMANN, 2002a, p. 57) ${ }^{13}$.

Em verdade, conquanto tomasse Karl Marx para si como exemplo de intelectual ("Ich nehme mal Marx") cujo pensamento poderia ser definido pela capacidade invulgar de comparar fenômenos heterogêneos em termos gerais por intermédio da construção conceitual (LUHMANN, 1987, p. 26-27) ${ }^{14}$, Luhmann não tinha em mente uma teoria da práxis, no sentido daquele filósofo alemão; ou a décima primeira tese sobre Feuerbach como uma espécie de mantra. Muito embora sua teoria conceitual da sociedade se

\footnotetext{
${ }^{11}$ No original: "Ein durchgehender Zug ist sicher mein Versuch, Distanz zu halten gegenüber solchen Phänomen, bei denen andere sich aufregen oder wo gewöhnlich normatives oder emotionales Engagement gefragt ist. Mein Hauptziel als Wissenschaftler ist die Verbesserung der soziologischen Beschreibung der Gesellschaft und nicht die Verbesserung der Gesellschaft. Das schließt natürlich nicht aus, daß man als ein anderer auftritt, wenn man am politischen oder am kirchlichen Leben oder auch an künstlerischen Veranstaltungen teilnimmt."
}

12 "Como ramo do ensino e da investigação, a sociologia tem de se poder fundar. Como disciplina científica não tem ainda, em grande parte, o carácter de disciplina. Certas "tendências" podem sobressair em análises duplas das publicações existentes. Mas falta uma concepção teórica global e conjunta, que reflicta a unidade da especialidade. Até pretensões que vão neste sentido são anunciadas apenas por alguns investigadores e só a custo - e, em seguida, fica-se quase sempre pelo anúncio" (LUHMANN, 2005, p. 71).

${ }^{13}$ Cf. ainda: "Die Soziologie steckt in einer Theoriekrise. Eine im ganzen recht erfolgreiche empirische Forschung hat unser Wissen vermehrt, hat aber nicht zur Bildung einer facheinheitlichen Theorie geführt. Als empirische Wissenschaft kann die Soziologie den Anspruch nicht aufgeben, ihre Aussagen an Hand von Daten zu überprüfen, die der Realität abgewonnen sind, wie immer alt oder neu die Schläuche sein mögen, in die man das Gewonnene abfüllt. Sie kann gerade mit diesem Prinzip jedoch die Besonderheit ihres Gegenstandsbereiches und ihre eigene Einheit als wissenschaftliche Disziplin nicht begründen. Die Resignation geht so weit, daß man dies gar nicht mehr versucht. Dieses Dilemma hat den Theoriebegriff selbst gespalten." (LUHMANN, 1991, p. 07). Tradução livre: "A sociologia se encontra em uma crise teórica. A pesquisa empírica, com bastante êxito, tem feito medrar o nosso conhecimento, mas não tem conduzido à formação de uma teoria unificada e específica, própria de sua disciplina. Como ciência empírica, a sociologia não pode prescindir da pretensão de comprovar suas afirmações mediante os dados obtidos da realidade, não importa quão velhos ou novos sejam os tubos nos quais se despeja o que já se verteu. No entanto, ela não pode, em razão deste princípio, fundamentar a natureza específica de sua competência e a sua unidade como disciplina científica. A resignação vai tão longe, que nem sequer há mais tentativa. Este dilema tem dividido o conceito mesmo de teoria."

14 “A resposta se insere nesse diálogo: Entrevistador: "Essa é uma definição tão incomum do intelectual ou da intelectualidade que eu gostaria de lhe pedir um exemplo. Luhmann: Eu tomo Karl Marx." (BACHUR, 2009, p. 09). 
pretendesse ainda mais radical que o marxismo mesmo ${ }^{15}$. Mas uma coisa seria o cientista social; outra, o político propriamente dito.

Nada obstante, ainda naquela mesma ocasião da ironia de Bonn, Luhmann veio a interceder em favor da eficácia política de sua teoria, a contrário senso: “Quer dizer, a teoria dos sistemas poderia ser politicamente eficaz nesse sentido [no que diz respeito a regimes totalitaristas ou terroristas], se ela critica, descobre e exibe as consequências de certas pretensões extremistas no campo da política ${ }^{16}$."

Para Horkheimer a verdadeira função social da filosofia é a crítica do status quo. Assumindo seriamente esse ponto de partida, a teoria crítica dos sistemas não é meramente tecnologia social, nem descrição sociológica extrínseca, nem autodescrição teórico-jurídica, mas um esforço eminentemente filosófico de crítica social. Para tal projeto de crítica, não há ponto fixo fora da sociedade; na verdade, a crítica tem de começar pelo referencial transcendente que transborda da própria imanência. [...] Uma vez que não existe um sujeito que paira por sobre a sociedade, não há posição disponível fora da sociedade a partir da qual as coisas possam ser claramente descritas [...] (FISCHER-LESCANO, 2010, p. 173-174).

\footnotetext{
15 "In an interview toward the end of his life, Luhmann stated: "It had always been clear to me that a thoroughly constructed conceptual theory of society would be much more radical and much more discomforting in its effects than narrowly focused criticisms - criticisms of capitalism for instance - could ever imagine. This is a remarkable pronouncement. Not only does Luhmann openly declare his radical intentions, but he explicity says that he intended his theory to be "much more radical and discomforting" - that is, more revolutionary -, than, well, Marxism." (MOELLERS, 2011, p. 04, grifo nosso). Cf. Thornhill (2013, p. 269).

${ }^{16}$ No original: "Das heißt, die Systemtheorie würde wirksam werden in dem Sinne, dass die Konsequenzen bestimmte, extreme der Annahme der Politik kritisiert, aufweist, aufdeckt behandelt.". Ainda a propósito de uma "teoria dos sistemas crítica": "In der sozialwissenschaftlichen Diskussion besteht seit langem der Verdacht, dass die Systemsoziologie Niklas Luhmanns ein Potenzial für die Entwicklung einer kritisch-emanzipativen Gesellschaftstheorie besitzt, das noch unausgeschöpft ist. [...] Mit der Suche nach theoretischen Anschlüssen geht die Frage nach einer Gesellschaftskritik einher, die die Kritische Systemtheorie zu entwickeln hat: Wie ist eine systemtheoretisch informierte, immanente Gesellschaftskritik möglich? Das kritische Potenzial der Systemtheorie kann zum einen in einer präzisen Deskription gesellschaftlicher Herrschaftsverhältnisse verortet werden. Indem die Systemtheorie als elaborierteste Selbstbeschreibung der bürgerlichen Gesellschaft das Instrumentarium für das Verständnis des an Tempo stets zulegenden Wandels der sozialen Strukturen zur Verfügung stellt, spielt sie eine Dimension ihres Kritikpotenzials aus, die in der Visibilisierung des Invisibilisierten besteht. [...] Die Systemtheorie der Weltgesellschaft artikuliert sich als kritische Theorie sozialer Systeme, wenn sie über eine bloß deskriptive Beschreibung der Strukturprobleme hinausgeht und die Gesellschaftsstrukturen einer Kritik unterzieht, die für postmaterialistische Theorien aktuellen Zuschnitts in vielfältiger Form produktiv anschlussfähig ist." (FISCHER-LESCANO, 2013, p. 7-14). Tradução dos autores: "No debate científico das ciências sociais persiste há muito a suspeita de que a sociologia sistêmica de Niklas Luhmann é dotada de um potencial, ainda não explorado, para o desenvolvimento de uma teoria da sociedade crítico-emancipatória. [...] Com a busca por conexões teóricas vem acompanhada a questão de uma crítica da sociedade, a qual a teoria dos sistemas crítica tem de desenvolver. Como é possível, de modo sistêmico-teórico, uma crítica informada, imanente, da sociedade? O potencial crítico da teoria dos sistemas se depara aí numa descrição precisa das relações sociais de domínio. A teoria dos sistemas, enquanto a mais elaborada autodescrição da sociedade burguesa, ao fornecer o instrumental para a compreensão do ritmo das mudanças sempre determinadas das estruturas sociais disponíveis, ela desempenha uma dimensão de seu potencial crítico, o qual se vislumbra na visibilitação do invisibilitado. [...] A teoria dos sistemas da sociedade mundial se articula como teoria dos sistemas sociais crítica ao transcender uma análise puramente descritiva dos problemas estruturais e ao submeter as estruturas sociais a uma crítica, a qual se depara produtivamente conectiva pelos atuais recortes das teorias pós-materialistas."
} 
Não surpreende, portanto, que a adoção de uma abordagem sociológica eminentemente autocrítica, distanciada e não engajada, por assim dizer, carente de uma utopia social, tenha lhe rendido ao longo da vida os mais variados rótulos partidaristas, não pouco pejorativos, tais como "tecnocrata social enregelado", "conservador de vanguarda", "taoísta ocidental", "homem sem qualidades", "homem sem televisão", "aquele que dialoga com o fichário" ${ }^{17} \ldots$

Demais disso - e aqui figura o segundo motivo para a sua recusa - , causou espécie, à época, a colocação decerto polêmica do homem fora da sociedade, do homem enquanto ambiente da sociedade. Na perspectiva sistêmica, o homem, em sendo concebido como endereço de processos de comunicação, consubstancia-se, antes, em um problema para a sociedade e vice-versa. Assim, a sociologia de Luhmann só podia parecer aos olhos de então como um tipo de heresia: “Um tal projeto teórico evoca a crítica humanista. Quem concebe a sociedade como algo outro que não uma comunidade de indivíduos, embarca em reflexões sobre o inumano, age de modo contraintuitivo, não se interessa pelo destino da humanidade" (FISCHER-LESCANO, 2013, p. 19). ${ }^{18}$

Esse "alheamento" não assume, no entanto, feição ontológica; trata-se de um "antihumanismo metodológico", para ficar aqui com o jargão conceitual de Habermas, insculpido em sua crítica ao livro "Soziale Systeme", segundo o próprio Luhmann, o "capítulo zero" de sua teoria da sociedade, que veio a público em 1984.

[...] "Si se considera al hombre como parte del entorno de la sociedad (en vez de como parte de la sociedad misma), ello cambiaría las premisas de todos los planteamientos de la tradición, $y$, por tanto, también las premisas del humanismo clásico." (288) Y a la inversa: "Quien se atenga a esas premisas y trate de defender con ellas um interés de lo "humano", no tendrá mas remedio que presentarse

\footnotetext{
${ }^{17}$ No original: "[...] kühler Sozialtechnokrat", "Avantgarde-Konservativer", "westlicher Taoist", "Mann ohne Eingeschaften", "Mann ohne Fernseher" und "der mit dem Zettelkasten kommuniziert." (RÜHL, 2008, p. 42). Cf. Hagen (2004/2005). O último apelido é digno de menção, pois faz uma referência jocosa à extraordinária força de trabalho de Luhmann, "a qual se baseou notoriamente na organização de um imenso fichário", o famoso Zettelkasten (LUHMANN, 2000, p. 153): “[...] un archivo conformado por un número superior a las cien mil fichas [...]. Desde las numerosas cajas que conforman este ficheiro, van saliendo los temas, los artículos y los libros, de tal manera que el autor llega a decir, em uma comentada entrevista, que su mayor trabajo es con la Zettelkasten, porque los libros se escriben solos" (LUHMANN, 2002c, p. 26).

${ }^{18}$ No original: "Ein solches Theoriedesign evoziert humanistische Kritik. Wer Gesellschaft anders denn als Zusammenschluss von Individualmenschen konzepiere, denke das A-Humane, agiere kontraintuitiv, interessiere sich nicht für menschlische Schiksal."
} 
como adversario de la pretensión de universalidade de la teoria de sistemas" (LUHMANN apud HABERMAS, 1985, p. 443; HABERMAS, 2000, p. 523) ${ }^{19}$.

\section{Revertendo as acusações provenientes de Frankfurt, Luhmann vislumbra que "a} teoria dos sistemas autopoiéticos poderia, de fato, ostentar o título Levando os Indivíduos a Sério, certamente de modo ainda mais sério que a nossa tradição humanista" (LUHMANN, 1992, p. 1422, itálico no original), ${ }^{20}$ precisamente porque não operaria com uma noção demasiado simplificada de indivíduo, o que é, na sua opinião, uma idiossincrasia da abordagem tradicional ${ }^{21}$.

Com muita paciência e alguma ironia, Luhmann refuta essas críticas, pois a teoria dos sistemas leva o "indivíduo" a sério precisamente porque o exclui da sociedade - e também da sociologia. O indivíduo não é então reduzido a um par de fórmulas, nem degradado à condição de máquina trivial, como é habitual na tradição das teorias sociais, as quais tinham o condão de saber o que ele seria em exatidão - de Zoon politikon [animal político, no sentido aristotélico] até lobo do próprio homem, de bom selvagem a aleijão desvalido - para a partir dessas certezas erigir seus esboços sociais. Luhmann elenca o indivíduo em relação ao ambiente da sociedade; ele é - entre outros - seu medium. Luhmann teve de sofrer por décadas da "irritação" dessa cefaleia, a qual - dito por ele assim de modo ambíguo -- deve ser capaz de acompanhar todo o seu pensamento (WERBER, 1998) $)^{22}$.

\footnotetext{
${ }^{19}$ Ver ainda nota de rodapé n 24 desse primeiro livro (1985, p. 443): “Luhmann acentúa "que para la tradición humanista el hombre estava dentro y no fuera del orden social. Se lo consideraba como ingrediente del orden social, como elemento de la sociedad misma. Se lo llamaba individuo porque era para la sociedad un elemento último non susceptible de ulterior decomposición"”".

${ }^{20}$ No original: "In fact, the theory of the autopoietic systems could bear the title Taking Individuals Seriously, certainly more seriously than our humanistic tradition. Taken as an individual, no human being can be part of any systems. Critics frequently miss this point." Cf. ainda no mesmo sentido: "In accordance with this unchallenged anthropocentric dogma of Western philosophy from Plato up and including the twentieth century, philosophy had always included ethical deliberations and pronouncements. [...] Therefore, anthropocentric philosophy generally entailed pronouncements about how to behave - either as individuals or as a collective. Luhmann's "radical antihumanism" is not limited to a redescription of reality in an non anthropocentric way. It includes a dismissal of human agency. [...] $A$ radically antihumanist theory tries to explain why anthropocentrism - having been abolished in cosmology, biology and psychology - now has to be abolished in social theory. Once this abolition has taken place, there is not much room left for traditional philosophical enquiries of a humanistic sort" (MOELLERS, 2011, p. 05-06).
}

21 "As an answer to the question why he omits the central self-reference of human beings, the concept of subject, Luhmann also [...] that with the omission of the concept of subject or of a general concept for human beings, his intention is to avoid or pretend that overall ethical claims are made on behalf of human beings or mankind. It is Luhmann's phobia against any ethical prescription. And there is that beautiful statement: "I know one single maxim in ethics, avoiding ethics". I share here Luhmann's opinion that it is indeed dangerous to define any prescription on behalf of mankind" (GUMBRECHT, 2012, p. 11). A propósito, há quase meio milênio Montaigne já ensaiava: "Na verdade, o homem é um sujeito maravilhosamente vão, diverso e ondulante: é árduo estabelecer sobre ele um julgamento constante e uniforme" (MONTAIGNE, 2010, p. 45).

22 "Obituário" escrito por ocasião da morte de Luhmann, ocorrida poucos dias antes. No original: "Mit größter Geduld und einiger Ironie verwies Luhmann diese Kritiker darauf, daß die Systemtheorie "den Menschen" gerade dadurch ernst nehme, daß sie ihn aus der Gesellschaft - und damit auch aus der Soziologie - auschließe. Denn der Mensch werde so nicht auf ein paar Formeln reduziert und zur Trivialmaschine degradiert, wie dies in der Tradition der 
Ao que consta, Habermas teria mal compreendido a tentativa luhmanniana de dissipação da problemática ontológica pela via da teoria dos sistemas sociais, cuja intervenção teria por efeito justamente a "de-ontologização da realidade" (LUHMANN, 2002b, p. 132; LUHMANN, 2005b, p. 37) ${ }^{23}$. Com o aumento da disseminação e recepção da teoria dos sistemas no âmbito mundial, não apenas a nível acadêmico ou sociológico, tem se reconhecido paulatinamente que Luhmann não era deliberadamente "nenhum conservador, nem anti-iluminista, tampouco reacionário, sobretudo" (REHBERG, 2005, p. $285-309)^{24}$.

A terceira razão suficiente, aqui no sentido de Leibniz, para a presença esmaecida da Teoria dos Sistemas no cenário acadêmico é possivelmente o grau sobremodo abstrato do artefato teórico. Luhmann, é bem verdade, nunca represou um descontentamento com a teoria hodierna. À sua crítica a um notório déficit teórico da sociologia no tocante à observação e descrição da sociedade contemporânea dita hipercomplexa e policontextural, sobrejaz menos a amplidão de temas que a teoria dos sistemas tem naturalmente por pretensão abarcar do que a empresa de construção conceitual luhmanniana de uma nova trama categorial, pois a realidade não se capta e entrevê, segundo ele, senão por meio de conceitos laboriosos. ${ }^{25}$ Somada a essa percepção de

Gesellschaftstheorien üblich ist, die immer genau zu wissen meinten, was er sei, vom zoon politikon bis zur wölfischen Bestie, vom guten Menschen bis zum hilflosen Mängelwesen, um auf diesen Gewißheiten ihre Sozialenwürfe zu bauen. Luhmann zählt den Menschen dagegen zur Umwelt der Gesellschaft. Er ist - wie manches andere - ihr Medium. Vom Kopfschmerz, unter dessen "Irritation" Luhmann Jahrzehnte zu leiden hatte, sagte er vieldeutig, daß er "all sein Denken muß begleiten können."

${ }^{23}$ No mesmo sentido: "One of Luhmann's most provocative self-designations was "radical antihumanist". This, of course, was not an indication of sympathy for satanically inhumane values, methods, or political programs, but an attempt to fundamentally "de-anthropologize" the description of society and of the world in general" (MOELLERS, 2011, p. 05).

${ }^{24}$ No original: “(...) kein Konservativer, kein Anti-Aufklärer, erst recht nicht reaktionär (...)”. Portanto, não é fortuito lembrar que, até pouco, entendia-se entre nós a teoria dos sistemas - com base em Ingeborg Maus e na vinculação feita por Habermas, em sua Teoria do Agir Comunicativo, da teoria luhmmaniana da legitimação ao decisionismo de Carl Schmitt - como proposta teórica "neoconservadora, posto Luhmman afirmar a não produção de legitimidade pelos processos que em si sejam legítimos" (LIMA, 1996, p. 97).

${ }^{25}$ "Im Anschluß an Saussure, Kelly und andere könnte man auch formulieren: Begriffe formieren den Realitätskontakt der Wissenschaft (und das heißt wie immer so auch hier: eingeschlossen den Kontakt mit ihrer eigenen Realität) als Differenzerfahrung. Und Differenzerfahrung ist Bedingung der Möglichkeit von Informationsgewinn und Informationsverarbeitung. Punkt für Punkt kann es Entsprechungen von Begriff und Realitätgeben, etwa zwischen dem Sinnbegriff und dem Phänomen Sinn, ohne das keine Menschenwelt bestehen könnte. Entscheidend ist jedoch, daß die Wissenschaft, Systeme bildend, übers olche Punkt-für-Punkt Entsprechungen hinausgeht; daß sie sich nicht darauf beschränkt, zu copieren, zu imitieren, widerzuspiegeln, zu repräsentieren; sondern daß sie Differenzerfahrungen und damit Informations gewinnung organisiert und dafür adequate Eigenkomplexität ausbildet" (LUHMANN, 1991, p. 13). 
insuficiência, as transformações das estruturas sociais implicariam necessariamente transformações das estruturas semânticas (LUHMANN, 1997, p. 866-1149), aqui compreendidas como o acervo cultural e conceitual da sociedade mobilizado em sua autoobservação e autodescrição.

Não se pode deixar de usar conceitos estabelecidos: política, opinião pública, reflexão, poder, amor, apenas para nomear alguns. Entretanto, esperam-se progressos do trabalho científico, e portanto transformações. Na medida em que tais situações situam-se níveis exigentes de teoria, depara-se sempre com a questão do que é melhor: continuar com as terminologias, embora seu significado se transforme, ou despachá-las e, com isso, renunciar às linhas de identificação com a tradição (LUHMANN, 2002a, p. 54).

Ao abordar ou descrever os fatos com pretensão de universalidade, as palavras da vida cotidiana não bastam. Devem ser construídos conceitos. Somente é possível observar o que se construiu com esses conceitos, isto é, outros conceitos e outras coisas. Por sua vez, os conceitos têm distinta qualidade científica, dependendo do emprego teórico, mas independentemente dele, pode-se afirmar que com conceitos distintos constroem-se mundos distintos (LUHMANN apud MÉLICH, 1996, p. 13).

De sorte que a crítica ao seu estilo lhe foi uma companheira inseparável. É de Habermas, por exemplo, a mescla de encômio e suspeita seguinte: "A teoria de Luhmann, hoje incomparável sob o aspecto de sua força de conceptualização, de sua imaginação teórica e de sua capacidade de elaboração, desperta, em todo caso, dúvidas na questão de saber se o preço de seu "ganho de abstração" não seria demasiado alto." (2000, p. 492). ${ }^{26}$ Há quem compreenda o caráter altamente abstrato da obra luhmanniana como, em verdade, uma terminologia sobremaneira imaginativa a serviço de uma estratégia sofisticada de automistificação ante certas inépcias da própria teoria, de modo a ocultá-las

Tradução livre: "Em referência a Saussure, Kelly e outros, poder-se-ia assim formular: conceitos conformam o contato da ciência com a realidade (e, como sempre, quer-se dizer também aqui: incluso o contato com sua própria realidade), como experiência da diferença. A experiência da diferença é condição de possibilidade de obtenção e processamento de informação. Ponto a ponto, pode haver correspondências entre conceito e realidade, algo ali entre o conceito e o fenômeno de sentido, sem o qual não poderia existir o mundo do indivíduo. É crucial, no entanto, para a ciência o criar sistemas que transcendam ponto a ponto tais correspondências; que não se limitem a copiar, imitar, refletir, representar; sem que organizem a experiência da diferença e com ela ganhe em informação; que forme, assim, uma adequada complexidade própria". Cf. ainda: "Progress in the development of sociological theory, specially in the direction of an adequate theory of modern society, depends on implausible certainties, which must be secured through protracted, conceptually controlled, theoretical work" ("Instead of a Preface to the English Edition" in LUHMANN, 1995, p. 44, ênfase no original).

${ }^{26}$ Veja-se ainda: "La teoría de Luhmann me parece súper abstracta (est oes: las ventajas previstas em la abstracción se transforma em perdidas debidas a la abstracción) y supercompleja (es decir: la sustancialidad y el ingenio de las argumentaciones no se producen gracias a la posición metodológica, sino a pesar de ella)" (HABERMAS, 1986, p. 126). 
(KJAER, 2006, p. 74). Decorreria diretamente desse estratagema a criação de um universo dito autônomo, consubstanciado por centenas de conceitos que teriam engendrado consequentemente, a necessidade premente de publicação de um dicionário específico, designado a desvelar ao mundo essa nova linguagem como que hierática (KRAUSE, 2001; BARALDI, 1997). Por outro lado, outros há a não enxergar engenhosidade qualquer, tampouco faceta alguma do que seja mesmo autêntico, profundo ou complexo em sua obra.

Por trás da fachada de uma imensa dificuldade e de um mecanismo complexo próprio de uma terminologia artística, amontoa-se meramente um punhado de sentenças singelas: o mundo é complexo, tudo se liga a tudo, o indivíduo suporta apenas um grau limitado de complexidade. A sugestão de originalidade absoluta é reforçada pela criação de uma teoria da linguagem acessível unicamente para "iniciados". E este caráter esotérico do jogo conceitual me parece ser a segunda razão para a ampla atenção conferida a Luhmann. [...] Tal como em Heidegger, mais cedo, e depois em Adorno, o virtuosismo da mestria do jargão em que consiste o discurso luhmânnico consubstancia o diferenciador de sua imanência. Quanto mais obscuro e ininteligível, tanto mais magistral ${ }^{27}$.

A suposta autoconversão da teoria dos sistemas numa espécie de "mística" de cepa dogmática como que a elevaria acima das possibilidades de exposição e exame, imunizando-a, por isso mesmo, contra toda crítica, a qual seria tanto possível quanto maior fosse o grau de familiaridade do crítico com a sua terminologia: para refutá-la ou até mesmo ressalvá-la, parece necessário fazê-lo, antes, no íntimo de sua própria linguagem. Embora tal atitude se assemelhe porventura à empreitada de certos teólogos modernos, ${ }^{28}$ é do próprio Hegel, a quem Luhmann é constantamente aparentado, a seguinte arguição de nulidade de uma crítica que se quer apenas a nível exógeno de um sistema a outro:

27 Disponível integralmente no sítio da revista Der Spiegel $\mathrm{n}^{\circ}$ 50/1984, de 10/12/1984 (pp. 184-190): http://www.spiegel.de/spiegel/print/d-13511556.html. Acesso em: 10 fev. 20178. No original: “Dabei steck thinter der Fassade ungeheurer Schwierigkeit und einem komplizierten Räderwerk artistischer Begrifflichkeit lediglich eine Handvoll simpler Sätze: Die Welt ist kompliziert, alles ist MIT allem verbunden, der Menscher trägt nur ein begrenztes Maß an Kompliziertheit. Die Suggestion absoluter Originalität wird verstärk tdurch die Schaffung einer Theoriesprache, die nur den "Eingeweihten" zugänglich ist. Und diese Esoterik des Begriffsspiels scheint mir der zweite Grundfür die öffentliche Aufmerksamkeit Luhmann gegenüber zu sein. [...] Ähnlich wie früher bei Heidegger, und danach bei Adorno, wird die Virtuosität der Jargonbeherrschung, das "Luhmannisch"-Reden, zum Erkennungs zeichen des "DazuGehörens". Je dunkler und unverständlicher, desto meisterhafter." Cf. Käsler (1984, p. 188-189); Kunczik e Zipfel (2005, p. 84).

${ }^{28}$ Quando afirmam que o "Deus bíblico nada tem a ver com a metafísica, uma invenção grega, e que apenas foi falsamente confundida com isto; isso significa, com outras palavras: nós não queremos nos submeter às regras da argumentação metafísica, nem nos sentir atingidos pela crítica à metafísica; antes queremos falar de Deus como nos convier" (TÜRCKE, 1993, p. 30, grifo nosso). 
Further, any refutation would have to come not from outside, that is, not proceed from assumptions lying outside the system and irrelevant to it. The system need only refuse to recognize those assumptions; the defect is such only for one who starts from such needs and requirements as are based on them. [...] The nerve, therefore of any external refutation consists solely in obstinately clinging to the opposite categories of these assumptions [...]. Effective refutation must infiltrate the opponent's stronghold and meet him in his own ground; there is no point in attacking him outside his territory and claiming jurisdiction where he is not. The only possible refutation [...] can only consist, therefore, in first acknowledging its standpoint as essencial and necessary and then raising it to a higher standpoint on the strength of its own resources (HEGEL, 2010, p. 512, grifo no original).

A impressão de uma nova sorte de dialeto do pensamento sociológico, isto é "o elevado adensamento textual" requerido à interpretação do sentido, advém sobretudo da importação, apropriação, renomeação e ressemantização, empreendidas por Luhmann, de conceitos firmados por outros pensadores - quase sempre não canônicos - das mais diversas ciências e modelos teóricos, acompanhados ainda de um movimento de transposição de termos das ciências naturais para as ciências humanas (insinuando-se aí algo do desejo positivista de precisão, ainda latente), e ora reapresentados ou propostos em meio ao contexto social. Esse caráter estilístico idiossincrático certamente tem engendrado as dificuldades inerentes à sua escrita, bem como consistido talvez no maior entrave à sua divulgação.

Entre outras correntes, foi influencidado pela teoria biológica da autopoiese, formulada pelos chilenos Humberto Maturana e Francisco Maturana, da teoria do cálculo de George Spencer-Brown, do construtivismo de Heinz von Foerster, dos modelos cibernéticos, da filosofia fenomenológica de Husserl, da teoria social de Émile Durkheim e Talcott Parsons (NEVES, 2013, p. 336).

[...] a obsessiva procura da inovação, um quadro de referências desconcertantes (extensíssimo, muito diversificado, leituras à revelia da "cartilha" consagrada, citações desconhecidas ou, simplesmente, desprezadas...), a preocupação constante de desenvolver uma perspectiva teórica global (mesmo quando os temas abordados parecem sugerir grande especifícidade e um estilo de abordagem mais "prático") e, suprema dificuldade, a exploração minuciosa de campos bibliográficos imensos, numa intenção interdisciplinar que deixa desconcertados mesmo os mais eruditos. [...] E há, ainda, a dificuldade da escrita propriamente dita, que está na razão directa da complexidade da teoria que pretende exprimir: um estilo muito pessoal e complexo, com criação de terminologia própria, recorrências sistemáticas (nem sempre evidentes), concatenação caótica de diversos planos de análise e a multiplicação torrencial das referências. Todos estes factores são razões de sobra que fazem de Luhmann um autor pouco "apetecível" dos agentes editoriais. Aos que se propõem conhecer o seu pensamento, é recomendável que tomem desde logo em devida 
conta as dificuldades que os esperam. A descrença que o próprio autor afirma quanto à originalidade da sua obra, ou a caracterização que dela faz como "simples descrição" da realidade social não passam de mera ilusão de facilidades..." (ESTEVES, 1993, p. 05-36).

Sepp Gumbrecht (2012, p. 03-04), como que desclassificando o caráter underground dos autores frequentemente citados por Luhmann, suspeita que todas essas referências externas a "supostas autoridades teóricas" seriam antes permutações do nome do próprio Luhmann. Autorreferências, portanto ${ }^{29}$.

Referindo-se ao monumento "teórico abstrato e, ao mesmo tempo, profundo, não ao conteúdo mesmo da teoria sistêmica", Ingeborg Maus disse certa vez ao Prof. Marcelo Neves - quem porventura mais tem contribuído para a difusão da teoria dos sistemas no Brasil - que Luhmann seria "o Hegel de nossos tempos e vai permanecer como um paradigma" (NEVES, 2004, p. 131). De fato, com alguma frequência e não sem estranheza, diz-se que Luhmann é igualmente ininteligível para os leitores nativos de países de língua alemã, assim como o era o próprio Hegel à sua época ${ }^{30}$. Em sua defesa, o próprio Luhmann retruca que, em face da possibilidade de uma apreensão não intermitente mas instantânea de texto e sentido, o sociólogo deve quedar apreensivo.

[...] então precisamente a compreensibilidade torna-se um problema e passa-se a desejar formas linguísticas que intermedeie uma medida suficiente de reserva e evitem uma compreensão demasiado rápida. Caso contrário, a sociologia cairia de fato em uma situação de predomínio na política das ideias, que lhe seria perniciosa. [...] Mas como se pode saber ou direcionar, por meio de sinais linguísticos, o que ele [o leitor] reatualiza a cada momento? Como se pode evitar que tradições conceituais ou preconceitos estranhos à teoria se imponham

\footnotetext{
${ }^{29}$ Finalmente, por que Luhmann seria um péssimo escritor e por que escreveria livros tão ruins, ver capítulo I.2 (“Why He wrote such bad books") de Moellers (2011, p. 11-15).

${ }^{30}$ Ora, quanto a Hegel, é famosa a anedota de que, após a publicação de seu célebre livro "Fenomenologia do Espírito", em 1807, os scholars alemães preferiram aguardar a tradução francesa na ânsia de assim poder talvez entendê-la. "In the realm of the great philosophy", nas palavras de Adorno, "Hegel is no doubt the only one with whom at times one literally does not know and cannot conclusevily determine what is being talked about, and with whom there is no guarantee that such a judgement is even possible" (ADORNO, 1993, p. 89). Adiante, Adorno (1993, p. 101102, grifo nosso) divisa o risco assumido por Hegel em contraposição à "covardia" ou indiferença de Wittgenstein: "Wittgenstein's maxim, "Whereof one cannot speak, thereof one must be silent", in which the extrem of positivism spills over to the gesture of reverent authoritarian authenticity, and which for that reason exerts a kind of intellectual mass suggestion, is utterly antiphilosophical. If philosophy can be defined at all, it is an effort to express things one cannot speak about, to help express the non-identical despite the fact that expressing it identifies it at the same

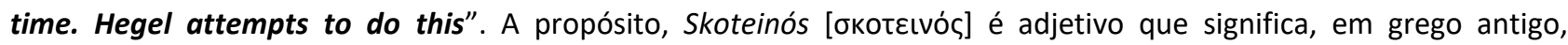
"repleto de escuridão" ou simplesmente "escuro".
} 
recorrentemente? Tudo isso exige um elevado adensamento textual" (LUHMANN, 2002a, p. 58) ${ }^{31}$.

A comparação com Hegel assume contornos ainda mais emblemáticos ao se ter notícia de que Luhmann fora agraciado em 1988 com o prestigioso "Hegel-Preis", prêmio concedido a cada triênio, desde 1970, a um reconhecido filósofo ou humanista, na cidade natal do próprio Hegel, cujo construto filosófico, sobretudo a lógica e a concepção de autorreferencialidade, Luhmann reverenciava e tinha em mais alta conta. As similaridades entre ambos se deparam ainda maiores ao se cotejar não somente estilos, mas ambições teóricas: indiscutivelmente, o ensaio luhmanniano de uma teoria da observação (de proporção catedralesca) ombreia e rivaliza com o intento hegeliano de uma descomunal construção teorética da realidade.

Sepp Gumbrecht coincidentemente chancela a associação de Maus ao asseverar que "Luhmann foi para o século XX o que Hegel tinha sido para o século XIX" (2010, p. 71), traçando alguns paralelos entre ambos, a fim de confirmar tal asserção ${ }^{32}$. Ademais, é sobremaneira expressivo que se tenha dispensado a Luhmann os mesmos predicados aparência de originalidade, jargonismo inane, automistificação, obscurantismo, esoterismo, charlatanismo etc. - uma vez e sempre condenados no próprio Hegel:

May also Hegel's philosophy of absolute nonsense (three-quarters cash and onequarter crazy notions) continue, as hitherto, to pass for unfanthomably profound wisdom, without anyone proposing as a motto to his works the words of Shakespeare: "Such stuff as madmen tongue and brain not", and, as an emblem at the head of theses works, a cuttle fish creating a cloud of obscurity around itself so

\footnotetext{
${ }^{31}$ Compare-se esse excerto à problemática, aventada por Adorno, que exsurge do desiderato de clareza: "Clarity can be demanded of all knowledge only when it has been determined that objects under investigation are free of all dynamic qualities that would cause them to elude the gaze that tries to capture and hole them unambiguously." (ADORNO, 1993, p. 98). Bordieu (2004, p. 68-73) participa da mesma opinião luhmanniana quanto à aparência "exotérica" da sociologia, que seria uma "ciência esotérica": "a iniciação é lenta e requer toda uma autêntica conversão de toda uma visão do mundo".

${ }^{32}$ Ver também: Gumbrecht (2012, p. 1-14. E ainda: “[A teoria dos sistemas] é um propósito teórico tão "totalitário" que torna compreensível uma afirmação como a do filósofo Robert Spaemann, para quem «se a teoria dos sistemas da Luhmann devesse ser comparada, numa comparação de teorias, com uma qualquer figura filosófica, uma tal figura só poderia ser a de Hegel». [...] O ar de família hegeliano que emana da teoria dos sistemas vem, pois, da sua capacidade de conceber uma forma de racionalidade, a racionalidade sistêmica, suficientemente flexível e universal para tornar inteligível o funcionamento dos mais diversos sistemas sociais, que vão da economia à arte, passando pelo direito, a política, a moral, a pedagogia e o sistema de ensino, a religião e mesmo o mais privado e íntimo dos sistemas que é o amor" (SANTOS, 2005, p. 10-11).
} 
that no one sees what it is, with the legend, mea-caligine-tutus. (SCHOPENHAUER, 2005, p. 81, grifo no original) ${ }^{33}$.

Em que pese a essas congeneridades, Luhmann houve por bem empreender, denunciam, uma tentativa de dissociação da teoria dos sistemas da tradição filosófica (a qual tachava de "alteuropa") ${ }^{34}$, máxime o idealismo alemão, do qual teria emergido; a teoria dos sistemas consubstanciar-se-ia, então, segundo seus críticos, num desenvolvimento ulterior do ideário de Kant, Fichte, Hegel e Husserl, ao apresentar soluções a um número crucial e até então insolúvel de problemas íntimos àquela tradição. Tal dissociação, acompanhada do aludido artifício de automistificação semântica, permite que a teoria dos sistemas assome ainda mais radicalmente enquanto novíssimo sistema teórico, aparentemente desprovido de precedentes.

É certo que Luhmann recusava com veemência a pecha de "filósofo"; referia-se a si mesmo, sempre que confrontado, como um "sociólogo" pura e simplesmente. ${ }^{35} \mathrm{E}$, em sua

\footnotetext{
${ }^{33}$ No entanto, Gérard Lebrun busca assentar, de modo decerto controverso, o sentido no qual somente seria possível o contraponto ou a rejeição de um sistema (no caso, o de Hegel), na medida em que não se lhe compreende o discurso a partir de pressupostos discursivos outros: "Se o sistema hegeliano é vulnerável, isso não se deve a seu dogmatismo (no sentido comum) nem a seu idealismo, ou ainda ao fato de que Hegel teria tratado superficialmente as ciências de sua época. [...] enquanto nos mantemos nessa posição [...], o sistema hegeliano permanece inexpugnável. Podemos muito bem acusá-lo de charlatanismo, mas não o refutaremos... [...]. Não se refuta um sistema da razão. Tudo o que se pode fazer, é reencontrar, bem ou mal e por subterfúgios, as escolhas léxicas nas quais ele se baseou e que traduzem, sem margem de dúvida, tomadas de posição eminentemente infra-racionais" (LEBRUN, 1988, p. 16, grifo nosso, itálico no original).

34 "In the fourth part, suggested by the title with the term "old Europe", I will try to assess how much Luhmann owes to old Europe, in spite of his own rhetoric, in which he always gets away from old Europe, i.e., the Western philosophical tradition. [...] Luhmann, at least this is what he explicitly affirms in his texts, wants to be anything but old Europe, i.e., he would rather be Heinz von Foerster or Fritz Heider, but not old Europe. But this external reference also turns into a strange self-reference, because anyone familiar with Luhmann's texts will clearly recognize that no other intellectual of his generation in Germany was so knowledgeable about the European philosophical tradition and used it as productively as he did" (GUMBRECHT, 2012, p. 02-04).
}

${ }^{35}$ Para mais sobre a persistência de Luhmann em se proclamar obstinadamente um sociólogo: "This is my main thesis: the void at the center of the theory does not enable the completion of the theory by one final thought, thus preserving its dynamism. This corresponds exactly to the reason, mentioned by the important philosopher Robert Spaermann in his eulogy, why Luhmann (whom he admires as a philosopher) defines himself so insistently, persistently and stubbornly as a sociologist, and not as a philosopher. Spaemann asks: isn't Luhmann, in fact, a philosopher, a modern Hegelian? (This question is, actually, valid and, to my joy, Spaemann mentioned Hegel too). And he continues: "This thesis would render him harmless" (that is, in fact, he is not a philosopher). 'Philosophy consists of final thoughts', to use an expression by Dieter Henrich. In this sense, philosophy seems naïve, because none of its thoughts has ever proven to be unsurpassable, but surpassing has always meant surpassing a final thought with another final thought that was understood as closure. Instead of final thought we could also say: Thought of the absolute. When I describe Luhmann's thinking as a challenge of philosophy, I do so for the following. reason: Luhmann has always consistently rejected any final thought". Later, Spaemann argues that this would be the reason why Luhmann ironically refers to himself as a sociologist, and by saying that Spaemann assumes rather optimistically that sociologists do not have any final thoughts" (GUMBRECHT, 2012, p. 11-12). 
concepção, para uma aparentemente incriada teoria dos sistemas, os idealismos kantiano e pós-kantiano teriam valor como que epifenomênico apenas.

Indeed, so successful was he in this strategy, that many present day system theoreticians appear to believe that Luhmann himself was responsible for the basic concepts of the systems theory. Consequently, many theoreticians also believe that systems theory is not embedded in any existing tradition - a problematic view, given that most system theoretical concepts derive almost directly from German idealism. [...] So, even though Luhmann "never engages in a painstaking and subtle reading of the classical canon of social theory", of the type undertaken by Derrida and Habermas, amongst others, one must agree with Habermas' statement that Luhmann's „UnternehmungsuchtAnschluß... an die Problemgeschichte der bewußtseinsphilosophie von Kant bis Husserl". Failure to acknowledge this heritage is becoming a major threat to the systems theory's future utility as a tool for social theoretical analysis. This is because, firstly, it has the consequence that many of Luhmann's disciples are only capable of defending systems theory from within, whereas they require a language bestowing a capacity to argue outside its own frame; secondly, because it provides a structural basis for theoretical fundamentalism, visible, for example, in the attempt to elevate the calculus of indication to a position where it supplies the basis of all other system theoretical concepts. Electing such a strategy for further theoretical development, however, undermines one of the strongest elements of system theory-namely, the stability its non-reductionist version possesses as a system, precisely because it is not based on any one element but on a whole range. One can always fetch another tool from the box if the first does not seem to fit. Such plurality also secures the theory against the kind of foundationalism which, as a radical anti-totalitarian, Luhmann sought to avoid (KJAER, 2006, p. 74, grifo no original) ${ }^{36}$.

\section{CONCLUSÃO}

A tríade de razões sobre as quais se ponderou acima contribuíram exponencialmente para com as possibilidades de inadmissão de Luhmann, em especial no Brasil, afetando, aliás, a disponibilidade de traduções de suas obras em português, cuja escassez (em contraste com as abundantes traduções em língua hispânica, à guisa de comparação) poderia ser elencada entre os motivos para uma tal irreceptibilidade, quando seria, antes, uma decorrência lógica demasiado coerente ${ }^{37}$.

\footnotetext{
${ }^{36} \mathrm{E}$ ainda: "Well, if we exclude from Luhmann's work all the multiple influences of old Europe, what remains as a difference, a claim to originality, is a negative diagnosis" (GUMBRECHT, 2012, p. 09-10). Outros autores apontam que é a comparação mesma entre Luhmann e Hegel uma das ilustrações da fenda entre a teoria sistêmica e a filosofia tradicional, pois aquela partilharia das aspirações dessa última e, a um só tempo, implicaria uma reflexão irônica acerca de suas limitações filosóficas (MOELLERS, 2011, p. 09).

${ }^{37}$ Para mais sobre os desafios da perspectiva sistêmica na América Latina, vide Birle et al. (2012, p. 07-16).
} 
Há quem situe, entre os porquês de sua descoberta ainda por se dar entre nós, o fato de que ele houve por bem eleger o recato da cidadezinha de Oerlinghausen - que "não favoreceu, seguramente, a projecção internacional" (ESTEVES, 1993, p. 05-36) como o meio ideal para seu trabalho de escultor teorético-sistêmico. No entanto, em tempos em que se pode ter, ao clique de um mouse, a notícia instantânea acerca de qualquer pensador e sua respectiva obra, mesmo do mais remoto rincão, essa tese não parece hoje minimamente convincente.

Por fim, conquanto sua obra se vislumbre desde a década de 1980, para alguns, entre os clássicos do pensamento europeu contemporâneo (COHN, 2013, p. 45-46), e, agora, prestes a ser celebrado os vintes anos de sua morte, que se deu precisamente em 06 de novembro de 1998, Luhmann ainda é por aqui um desconhecido ou, no melhor dos casos, um retardatário da desproporcional potência crítica de seus adversários festejados. Assim, resta dimensionar o que seria, então, mais deplorável a uma obra ou autor: se a sua recepção sem crítica ou se uma crítica que não é senão estorvo à sua recepção. Mas o dilema assoma talvez supérfluo ao se reconhecer, afinal, que dificilmente a obra de contemporâneos é o vetor mais importante na consolidação da educação de uma contemporaneidade em formação. Quase sempre não o é.

À guisa de conclusão, talvez seja proveitosa a transcrição do trecho final da já citada entrevista concedida a Ulrich Boehm, em 1973, tão só para se ter uma ideia de como Luhmann reagia de modo bem humorado a uma fortuna crítica a cujo infortúnio, já àquela época provavelmente, havia então se habituado: "Ulrich Boehm: - Professor Luhmann, quais críticas à sua Teoria dos Sistemas o Sr. mais teme? Luhmann: - As estúpidas”"38.

\footnotetext{
${ }^{38}$ No original: "Ulrich Boehm: Herr Professor Luhmann, welche Kritiker ihrer System theorie fürchten Sie am meisten? Luhmann: Die Dummen". E ainda em se tratando de anedotas em que figuram personalidades proeminentes (até para que se tenha deles uma impressão menos antipática), o bom humor de Luhmann, ao que consta, era uma constante em seu dia-a-dia: "As we know, Luhmann had na administrative career until he was forty-years old. As a consequence, the management of the Bielefeld University felt a certain respect for Niklas Luhmann. Their employees had idea of his academic importance, but they knew that he was knowledgeable about administration processes. And as all German professors, particularly in the miserly state of Nordrhein-Westfalen governed by the Social-Democratic Party (SPD), Luhmann had to fill out a list by the end of every month justifying all long distance calls he had made that had cost over 1.25 German Marks, or whatever the exact amount was. For thirteen years Luhmann, in his very clear handwriting, wrote the letters k.A. in the corresponding field [acronym for keine Angabe, i.e., nothing to declare]. This was accepted for thirteen years, but then a new administrator was hired by the "phone call control agency" and he did not know that Luhmann had had an administrative career. He then asked at the department office what the abbreviation "k.A." meant, because he wanted to learn its meaning. Luhmann told him it meant "keine Ahnung" [I
} 


\section{REFERÊNCIAS}

ADORNO, Theodor. "Skoteinós, or how to read Hegel". In: Hegel: Three Studies. Translation by Shierry Weber Nicholsen. Massachusetts Institute of Technology, 1993.

BACHUR, João Paulo. Distanciamento e crítica: limites e possibilidades da teoria dos sistemas de Niklas Luhmann. Tese apresentada ao Programa de Pós-Graduação do Departamento de Ciência Política da Faculdade de Filosofia, Letras e Ciências Humanas da Universidade de São Paulo, para obtenção do título d Doutor em Ciência Política. São Paulo, 2009.

BARALDI, Claudio et al. GLU: Glossar zu Niklas Luhmanns Theorie Sozialer Systeme. Frankfurt/M, Suhrkamp, 1997.

BIRLE, Peter et al. Zur Einführung - Luhmann und Lateinamerika. In: Durch Luhmanns Brille: Herausforderung an Politik und Recht in Lateinamerika und in der Weltgesellschaft. Springer VS, Wiesbaden, 2012.

BORCH, Christian. Niklas Luhmann (Key Sociologists). London and New York: Routledge, 2011.

BOURDIEU, Pierre. Coisas ditas. São Paulo: Brasiliense, 2004.

COHN, Gabriel. Max Weber: muito além do Estado Nacional. In: ALMEIDA, Jorge de; BADER, Wolfgang (Org.). $\mathbf{O}$ pensamento alemão no século $\mathbf{X X}$ : grandes protagonistas e recepção das obras no Brasil São Paulo: Cosac Naify, 2013, volume I. p. 28-46.

ESTEVES, João Pisarra. Uma Apresentação. In: LUHMANN, Niklas. A improbabilidade da Comunicação. Lisboa: Vega-Passagens, 1993. p. 05-36.

FERRAZ JR., Tércio Sampaio. Apresentação. In: LUHMANN, Niklas. Legitimação pelo procedimento. Tradução Maria da Conceição Corte-Real. Brasília: Ed. Universidade de Brasília, 1980.

FISCHER-LESCANO, Andreas. A Teoria Crítica dos Sistemas da Escola de Frankfurt. Tradução de Rurión Melo. Revista Novos Estudos - CEBRAP, n. 86, p. 163-177, mar. 2010.

FISCHER-LESCANO, Andreas. Systemtheorie als kritische Gesellschafttheorie. In: AMSTUTZ, Marc; FISCHER-LESCANO, Andreas (Hg.) Kritische Systemtheorie: Zur Evolution einer normativen Theorie. Bielefeld, Transcript, 1. Aufl., 2013, s. 13-30.

GUMBRECHT, Hans Ulrich. "Old Europe" and "the Sociologist": How does Niklas Luhmann's theory relate to philosophical tradition?. Revista da Associação dos Programas de PósGraduação em Comunicação - E-compós, Brasília, v. 15, n. 3, p. 1-14, set./dez. 2012. 
HABERMAS, Jürgen. El discurso filosófico de la modernidad. Madrid, Taurus, 1985.

HABERMAS, Jürgen. 0 discurso filosófico da modernidade: doze lições. Tradução de Luiz Sérgio Repa e Rodnei Nascimento. São Paulo: Martins Fontes, 2000.

HAGEN, Wolfgang (Hrsg.). Warum haben Sie keinen Fernseher, Herr Luhmann? Letzte Gespräche mit Niklas Luhmann. Kulturverlag Kadmos, Berlin, 2004/2005.

HEGEL, Georg Wilhelm Friedrich. The Science of Logic. Cambrigde University Press, 2010.

KJAER, Poul F. Systems in Context: On the Outcome of the Habermas/Luhmann-Debate. Ancilla Juris, 2006.

KÄSLER, Dick. Soziologie: Flug über den Wolken. Der Spiegel, 50/1984, p. 184-190, 10.12.1984.

KRAUSE, Detlef. Luhmann-Lexikon: eine Ein-führung in das Gesamtwerk von Niklas Luhmann. Stuttgart: Lucius \& Lucius, ,2001.

KUNCZIK Michael; ZIPFEL, Astrid. Publizistik: ein Studienbuch, Böhlau UTB, Stuttgart, 2005.

LEBRUN, Gérard. O Avesso da Dialética. São Paulo: Companhia das Letras, 1988.

LÉVI-STRAUSS, Claude. Tristes trópicos. Tradução de Wilson Martins. Revisão de LéviStrauss. São Paulo: Anhembi Limitada, 1957.

LIMA, Martônio Mont'alverne Barreto. Justiça constitucional e democracia: perspectivas para o papel do Poder Judiciário. Revista da Procuradoria Geral da República, São Paulo, v. 8, 1996.

LUHMANN, Niklas. Niklas Luhmann im Gespräch mit Ulrich Boehm über Systemtheorie. Uniaudimax, Sendung 28.08.1973, Kamera Bernd Maus.

LUHMANN, Niklas. Ich nehme mal Karl Marx. Interview mit Walter von Rossum. In: BAECKER, Dick; STANITZEK, Georg (Ed.). Archimedes und Wir. Interviews mit Niklas Luhmann. Berlin: Merve, 1987.

LUHMANN, Niklas. Soziale System: Grundriß einer allgemeiner Theorie. Frankfurt am Main: Suhrkamp-Taschenbuch Wissenschaft, 1991, 4. Aufklage.

LUHMANN, Niklas. Operational Closure and Structural Coupling: The differentiation of the legal system. Cardozo Law Review, 1992a, vol. 13.

LUHMANN, Niklas. Die Selbstbeobachtung des Systems: Ein Gespräch mit dem Soziologen Niklas Luhmann. Von BREUER, Ingeborg. Frankfurter Rundschau, FR, vom 5.12.1992b, s. ZB2.

LUHMANN, Niklas. Teoria de la sociedade y pedagogía. Barcelona: Paidós Educador, 1996.

LUHMANN, Niklas. Die Gesellschaft der Gesellschaft. Frankfurt am Main, Suhrkamp, 1997.

LUHMANN, Niklas. Social Systems. Translated by John Berdnarz, Jr. with Dick Baecker. Standford: Standford University Press, 1995. 
LUHMANN, Niklas. O paradoxo dos direitos humanos e três formas de seu desdobramento. Tradução de Paulo Antônio de Menezes Albuquerque e Ricardo Henrique Arruda de Paula. Themis, Fortaleza, v. 3, n. 1. , 2000.

LUHMANN, Niklas. Ciência incompreensível: problemas de uma linguagem própria à teoria (tradução do original por Leopoldo Waizbort. Revista Novos Estudos CEBRAP, n. 63, jul. 2002a.

LUHMANN, Niklas. Theories of Distinction. Stanford, CA: Stanford University Press, $2002 \mathrm{~b}$.

LUHMANN, Niklas. El derecho de la sociedad. Traducción de Javier Torres Nafarrate). México DF: Universidad Iberoamericana, 2002c.

LUHMANN, Niklas. Sociologia como teoria dos sistemas sociais. In: SANTOS, João Manuel (Org.). O Pensamento de Niklas Luhmann. Universidade da Beira Interior: Lusosofia Press, 2005, p. 371.

LUHMANN, Niklas. Soziologische Aufklärung 5: Konstruktivistische Perspektiven. Wiesbaden: VS Verlag, 2005b.

MÉLICH, J. Introdución: El Laberinto de la Teoria de la Sociedad. In: LUHMANN, Niklas. Teoria de sociedad y pedagogia. Barcelona: Paidós Educador, 1996.

MOELLER, Hans-Georg. The radical Luhmann. New York: Columbia University Press, 2011.

MONTAIGNE, Michel. Os Ensaios: uma seleção. Organização M. A. Screech. Tradução e notas de Rosa Freire de Aguiar. São Paulo: Companhia das Letras, 2010.

MÜLLER, Friedrich. Elementos para a renovação de um pensamento jurídico materialista: constituição, sociedade e democracia". In: LIMA, Martônio Mont'Alverne Barreto; BELLO, Enzo (Coord.). Direito e Marxismo. Rio de Janeiro: Lumen Juris, 2010.

NEVES, Marcelo. A Teoria dos Sistemas Sociais de Niklas Luhmman: entrevista com Marcelo Neves. Revista Plural Sociologia, USP, São Paulo, $2^{\circ}$ semestre 2004.

NEVES, Marcelo. Transconstitucionalismo. São Paulo: Martins Fontes, 2009.

NEVES, Marcelo. Entre Têmis e Leviatã: uma relação difícil: o Estado Democrático de Direito a partir e além de Luhmann e Habermas. São Paulo: Martins Fontes, 2012.

NEVES, Marcelo. Niklas Luhmann: "eu vejo o que tu não vês". In: O Pensamento Alemão no século XX: Grandes protagonistas e recepção das obras no Brasil. Vol. 1. 1ed. São Paulo: Cosac Naify Portátil, 2013.

NIETZSCHE, Friedrich. Sämtliche Briefe. Vol. 6. München: DTV/de Gruyter, 1986.

NIETZSCHE, Friedrich. Escritos sobre educação. Tradução de Noeli Correia de Melo Sobrinho. São Paulo: Edições Loyola, 2003.

NIETZSCHE, Friedrich. Além do bem e do mal: prelúdio a uma filosofia do futuro. São Paulo: Companhia das Letras, 2005.

PHILIPPOPOULOS-MIHALOPOULOS, Andreas; COUR, Anders la. Introduction: Luhmann Encountered. In: PHILIPPOPOULOS-MIHALOPOULOS, Andreas; COUR, Anders la (Coord.). Luhmann Observed: Radical Theoretical Encounters. Basingstoke: Palgrave, 2013. 
REHBERG, Karl-Siegbert. Konservativismus in postmodernen Zeiten: Niklas Luhmann. In: RUNKEL, Gunter; BURKART, Günter (Hrsg.): Funktionssysteme der Gesellschaft: Beiträge zur Systemtheorie von Niklas Luhmann. Wiesbaden: VS, 2005.

RÜHL, Manfred. Kommunikations Kulturen der Weltgesellschaft: Theorie der Kommunikationswissenschaft. Springer VS, 2008.

SANTOS, José Manuel. Apresentação. In: SANTOS, João Manuel (Org.). O Pensamento de Niklas Luhmann. Universidade da Beira Interior: Lusosofia Press, 2005.

SCHOPENHAUER, Arthur. Philosophical writings: Arthur Schopenhauer. Ed. by Wolfgang Schirmacher. German Library, vol. 27.

THORNHILL, Christopher. Luhmann and Marx: Social Theory and Social Freedom: Social Theory and Social Freedom. In: PHILIPPOPOULOS-MIHALOPOULOS, Andreas; COUR, Anders la (Coord.). Luhmann Observed: Radical Theoretical Encounters. Basingstoke: Palgrave, 2013.

WERBER, Niels. Der Mensch als Umwelt der Gesellschaft. In: Tages-Anzeiger Zürich vom 12.11.1998. 